\title{
Online Employer Reviews as a Data Source: A Systematic Literature Review
}

\author{
Christoph E. Höllig \\ Technical University of Munich \\ christoph.hoellig@tum.de
}

\begin{abstract}
Employees generate a rich source of information by publishing employer reviews online. However, researchers seeking to harness employer review data face an elusive body of research to build on. Therefore, I present a systematic, multidisciplinary literature review, presenting the information obtained from employer reviews, the research topics covered, and the data sources used. My analysis of 28 peerreviewed journal articles shows that studies extract insider knowledge, information on employee satisfaction and changes in employee satisfaction, insights into workplace culture, and linguistic style from employer reviews. Studies do so to predict firm performance, to explore employee satisfaction factors, and to uncover the linguistic style of employer reviews. Glassdoor is the primary data source used. Finally, I identify five avenues for further research.
\end{abstract}

\section{Introduction}

Nowadays, employer reviews are available in large volumes and from various providers. For instance, the employer review website Glassdoor reported a database of 55 million employer reviews, CEO approval ratings, salary reports, and other job insights in May 2020 [15]. The emergence of online employer reviews, as a unique type of user-generated content, is likely to provide new research opportunities beyond the work undertaken so far concerning online reviews of customers on products or services [41]. Harnessing these opportunities requires that researchers can identify the research topics addressable with employer review data and the information that can be extracted from these reviews' content. However, initial studies that may provide such insight are scattered across several disciplines and are thus challenging to survey.

Therefore, to facilitate the exploitation of online employer reviews, I conduct a systematic literature review (SLR) of the research that has so far been done utilizing employer review data and structure it into distinct concepts [see 47]. I pursue three research objectives. First, to identify the research topics addressable using employer review data. Second, to reveal the information extractable from employer reviews as well as how to do so. Finally, to spot relevant research gaps to stimulate future research using employer reviews.

\section{Theoretical Background}

For this study, I define online employer reviews as employee-generated employer evaluations or judgments posted on third-party websites [adapted from 31]. Employer reviews reflect the selected beliefs that current and former employees hold about their employer [adapted from 5]. Employees develop these beliefs through various experiences with their employer [see, e.g., 26].

Employer reviews' content is typically guided by a pre-defined questionnaire and comprises quantitative ratings on five-point Likert scales as well as openended text comments about the employer. For instance, the employer review website Kununu prompts reviewers to rate and comment on several individual aspects such as company culture and answer three brief questions on their employer's pros, cons, and opportunities for improvement.

Employer review websites operate comparably to product or service review websites such as Amazon, TripAdvisor, or Yelp, but invite employees instead of customers to generate content. So far, the emergence of product and service reviews has produced an extensive body of research [see, e.g., 21]. Since employer reviews likely provide rich information beyond that obtained from product and service reviews [see 41], employer reviews open up a rich and novel field of research.

\section{Methodology}

I conducted my SLR following the suggestions of Briner and Denyer [4]. First, I clearly defined my research objective. Second, I defined the literature databases as well as search terms relevant for my 
review. Third, I retrieved publications from the previously defined databases and merged the results. Fourth, I sifted through the identified publications and applied inclusion and exclusion criteria. Finally, I performed my review, i.e., conducted a full-text analysis with the remaining publications and synthesized my results into distinct concepts [see 47].

My literature review had the following objective: to identify studies that use online employer reviews as a data source to reveal the information obtained, the way the information was obtained, the research topics covered, and the data sources used. Thus, I only sought to identify studies that used, for instance, Glassdoor's employer reviews, but not other job information offered by employer reviews websites such as salary reports or interview experience reports.

To identify the overall body of research, I did not limit my review to a single discipline. Rather, I searched publication databases covering a multitude of disciplines: Scopus, EBSCOhost, ScienceDirect, ProQuest, Web of Science, ACM Digital Library, AIS eLibrary, and Google Scholar. To search those databases, I defined the following search string and adapted it to the syntax of the respective publication database:

("employer reviews" OR "online employer reviews" OR "online employee reviews" OR "employer review data" OR "employee review data" OR "glassdoor reviews") OR ("employee reviews AND "review data")

I used the search string for all fields (including title, abstract, keywords, and full text). However, I limited the database searches to full-text articles written in English. Further, to ensure consistency throughout my search phase and the associated repeated database searches, I only considered publications issued or in press before 1 January 2020. My search process yielded 53 results for Scopus, 49 results for EBSCOhost (Academic Journals, Journals, and Conference Materials), 72 results for ScienceDirect, three results for ProQuest, nine results for Web of Science, five results for ACM Digital Library, 12 result for AIS eLibrary, and 454 results on Google Scholar. In summary, my database search yielded 657 results. After eliminating duplicate entries based on the publications' titles, 569 publications remained.

Next, I screened titles, abstracts, keywords, and, if necessary, each publication's full text. Following my literature review's objective, I only retained publications that utilize actual online employer reviews as a data source in their studies. In this vein, 63 publications remained: eight conference proceedings, three dissertations, 29 working papers (e.g., published on SSRN), and 23 journal articles. To

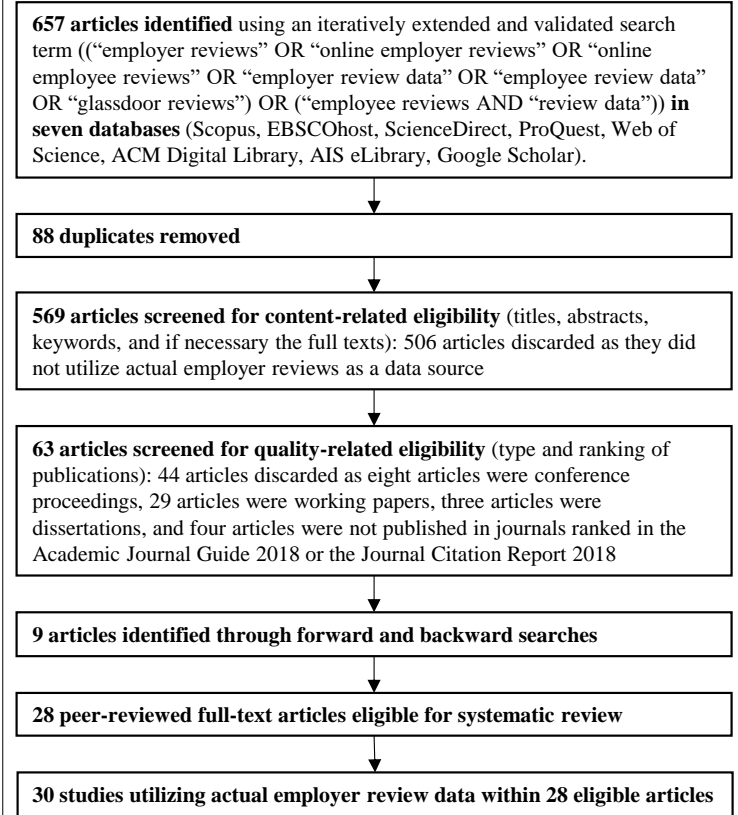

\section{Figure 1. Search process}

guarantee each publication's quality, I discarded publications not included in the Academic Journal Guide (AJG) 2018 by the Association of Business Schools or the Journal Citation Report (JCR) 2018 by Thomson Reuters. 19 peer-reviewed journal articles remained. Subsequently, to exhaust the relevant literature, I followed the citation trails that lead to other contributions. More specifically, I conducted forward and backward searches to identify publications missed with my search strategy. Forward and backward searches yielded another nine peerreviewed journal articles relevant to my review. Figure 1 provides an overview of my review's search process.

My review set comprises 28 peer-reviewed journal articles published between 2015 and 2020. The articles were published in journals with a JCR 2018 Journal Impact Factor between 0.876 and 9.360. I also coded the journals' discipline using the categories indicated by AJG and JCR. My review set covers nine disciplines, whereby most articles were published in the Management, Finance \& Accounting, and Marketing fields. For an overview of years and disciplines, refer to Figure 2.

The full-text analysis of the articles in my review set indicated that none of the articles split into multiple studies $[6,25,48]$ utilized employer reviews in more than one study. However, I noted that two articles do not make a distinction between individual studies, but still present several conceptually distinct analyses [19, 42]. Therefore, I counted them into more than one concept when synthesizing the results of my full-text analysis into distinct concepts [see 47]. However, I did 


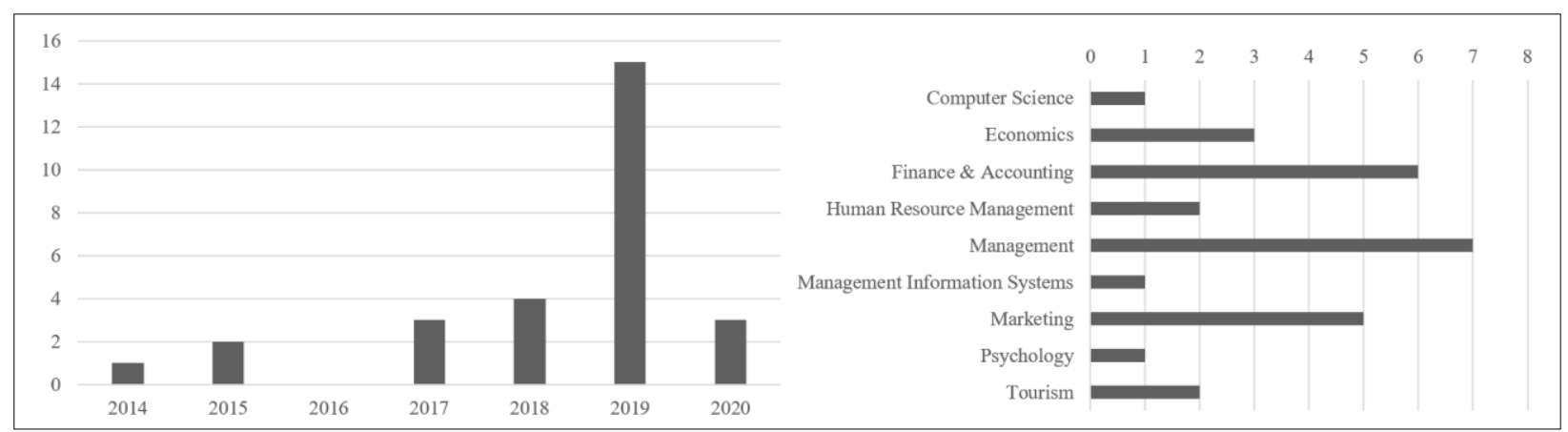

Figure 2. Years and disciplines covered with the SLR

not count determinant analyses [16, 17] as separate studies, as they were not within the actual scope of the articles and thus may not be subject to the same rigor as the articles' main analyses.

\section{Results}

Table 1 provides an overview of the concepts and their frequencies resulting from the analysis of the 28 peer-reviewed articles in my review set. I present my findings in more detail in the following.

Table 1. Frequencies of SLR concepts

\begin{tabular}{|c|c|c|}
\hline Concept & Frequency & Percent \\
\hline \multicolumn{3}{|c|}{ Research Topic } \\
\hline Predict firm performance & 10 & $33.33 \%$ \\
\hline $\begin{array}{l}\text { Explore factors of employee } \\
\text { satisfaction }\end{array}$ & 14 & $46.67 \%$ \\
\hline $\begin{array}{l}\text { Uncover the linguistic style of } \\
\text { employer reviews }\end{array}$ & 3 & $10.00 \%$ \\
\hline Other & 3 & $10.00 \%$ \\
\hline & $n=30$ & $100.00 \%$ \\
\hline \multicolumn{3}{|c|}{ Text Mining Techniques } \\
\hline Topic modeling & 4 & $25.00 \%$ \\
\hline Dictionary-based text analysis & 5 & $31.25 \%$ \\
\hline Data-mining software & 4 & $25.00 \%$ \\
\hline Individual word frequencies & 2 & $12.50 \%$ \\
\hline Other & 1 & $6.25 \%$ \\
\hline & $\mathrm{n}=16$ & $100.00 \%$ \\
\hline \multicolumn{3}{|c|}{ Data Sources } \\
\hline Glass & 24 & $85.71 \%$ \\
\hline Indee & 2 & $7.14 \%$ \\
\hline Jobplanet & 1 & $3.57 \%$ \\
\hline Kununu & 1 & $3.57 \%$ \\
\hline & $\mathrm{n}=28$ & $100.00 \%$ \\
\hline
\end{tabular}

\subsection{Research Topics}

4.1.1. Predict firm performance. Ten studies used information derived from online employer reviews to predict firm performance. Three different types of information can be obtained from employer reviews to estimate firm performance. First, employer reviews provide information about employee satisfaction and changes in employee satisfaction. Against this backdrop, employee satisfaction was positively correlated to firm performance with respect to return on assets (ROA) [19, 29, 42, 45], Tobin's Q [19, 45], operating margin [29], and revenue per employee [29]. Further, changes in employee satisfaction (i.e., employee satisfaction trajectories) of employees with customer contact correlated positively with customer satisfaction [48]. Moreover, quarterly changes in employee satisfaction of current (vs. former) employees and employees working in headquarter states predicted stock returns one-quarter-ahead [16].

Although Green et al. used information on employee satisfaction from employer reviews, their findings touch on the second type of information that can be obtained from employer reviews to estimate firm performance [16]: insider knowledge. For instance, Glassdoor's employer reviews include a survey of employees' opinions on their employer's six-month business outlook (positive, neutral, or negative). Specifically, employees are prompted with the following question: "In the next six months do you think your company will perform better, worse, or remain the same?" [17, p. 92]. This business outlook indicator was shown to predict future operating performance as indicated by ROA and various other performance components (sales growth, cost of goods sold, R\&D expenditures, and inventory turnover) [18]. Business outlook was more effective in estimating functional performance indicators if provided by employees from the same functional area (e.g., sales employees' outlook predicting sales growth) [18]. Further, business outlook was associated with a wide range of indicators for future corporate disclosures, including key income statement information, transitory reporting items (e.g., restructuring charges), earnings surprises, and management forecast news [17].

The third type of information that can be obtained from employer reviews to estimate firm performance is insights into workplace culture. Corritore et al. used employer reviews to capture two distinct types of cultural heterogeneity, namely interpersonal and intrapersonal heterogeneity [8]. Interpersonal heterogeneity describes the "misalignment in cultural perceptions among the individuals who make up the 
organization" [8, p. 8]. Intrapersonal heterogeneity describes "the breadth of cultural beliefs to which those individuals subscribe" $[8$, p. 8]. While interpersonal heterogeneity was negatively related to ROA as a proxy for a firm's capacity for efficient execution, intrapersonal heterogeneity was positively related to Tobin's Q and patent output, as a proxy for a firm's capacity for recombinant innovation and creativity [8]. Finally, Au et al. used employer reviews to gain information about the extent of companies' employee flexibility, defined as “employees' ability to react and respond to unexpected changes in the firm's environment" [2, p. 1]. Employee flexibility was positively associated with stock returns for firms exposed to external risk [2].

\subsubsection{Explore factors of employee satisfaction.} Rather than utilizing employee satisfaction information derived from employer reviews as an independent variable, 14 studies used this information as a dependent variable and explored employee satisfaction factors. Of these studies, six studies explored the role of factors derived from nonemployer review data on employee satisfaction. In this context, four distinct types of information from external data sources were utilized to estimate employee satisfaction. First, studies estimated employee satisfaction based on organizations' structures. Companies with demographically diverse boards were more likely to implement progressive management programs well received by employees [9]. Moreover, family firms' employees reported higher satisfaction in their employer reviews (also concerning individual aspects such as work/life balance) than employees of non-family firms [19].

Second, two studies estimated employee satisfaction based on organizations' workplace culture. Companies that emphasize (vs. do not emphasize) adaptability (e.g., act quickly, seize opportunities) with their culture received higher overall satisfaction ratings in their employer reviews [32]. Furthermore, Fortune 500 companies that maintain a growth (vs. fixed) mindset received higher culture and value ratings in their employer reviews [6].

Third, one study estimated employee satisfaction based on organizations' financials. Employees in financially constrained (vs. non-constrained) companies reported lower satisfaction ratings (as indicated by overall ratings and individual ratings of work/life balance, senior management, and career opportunities) [23].

Finally, one study estimated employee satisfaction based on policies in a Difference-in-Differences setting [44]. In detail, a state-level minimum wage increase positively affected newly hired employees' satisfaction, but negatively affected the satisfaction of senior and high-ranking (i.e., department managers) employees [44].

Moreover, eight studies explored the role of factors derived from employer review data on employee satisfaction. In this vein, studies in this group utilized various variables derived from reviews' (textual) content, including pro and con comments, as well as individual ratings and information on the tenure of reviewing employees (former vs. current employees). I provide an overview of text mining techniques used to extract information from employer reviews' textual content in the next section. Moro et al. demonstrated the most important satisfaction factors for IT employees based on a support vector machine (SVM) model [30]. Further, Jung and Suh identified 30 topics (i.e., job satisfaction factors), such as "organizational culture" via topic modeling [24]. Subsequently, they demonstrated that the sentiment and importance of these job satisfaction factors differ between industries, companies, years, and current and former employees [24]. Finally, they identified the most important factors for reviews' overall quantitative ratings in a dominance and correspondence analysis [24].

Stamolampros et al. employed several analyses to explore employee satisfaction factors in tourism and hospitality firms [42]. In detail, the authors examined the influence of individual ratings on reviews' overall rating [42]. In accordance with the findings of Jung and Suh, senior leadership was among the most important factors [24]. However, while Jung and Suh identified compensation and benefits as the second most important factor, compensation and benefits were least important for overall rating in the analysis of Stamolampros et al. [24, 42]. Stamolampros et al. further demonstrated that employees who choose to leave a firm are likely to be most dissatisfied, that male employees are more satisfied than female employees, and that companies with higher revenue also achieve higher employee satisfaction [42]. Further, the authors showed that an employee's likelihood of leaving a company is affected by culture values, senior leadership, and career opportunities. Finally, the authors also explored the textual contents of their employer review data set via topic modeling and identify 20 job satisfaction/dissatisfaction factors that differ in their prevalence according to reviews' overall rating [42]. Correspondingly, also Stamolampros et al. identified employee satisfaction factors in the tourism and hospitality industry using topic modeling [41]. The authors identified ten factors and demonstrated how the prevalence of these factors varies across subindustries. 
Finally, one study took another approach and did neither explore reviews' textual content, nor reviews' overall ratings, but utilized that employees also indicate whether they would recommend their employer through their Glassdoor reviews (yes vs. no). In this vein, Saini and Jawahar showed a positive impact of reviews' individual ratings as well as Universum's Top 100 employer ranking on employees' recommendations [38]. Further, by examining the interaction with employees' characteristics, the authors showed, for instance, that work/life balance is more important for part-time employees than for full-time employees [38].

Instead of identifying satisfaction factors at the review level, three studies compared satisfaction factors at the company level. In this vein, qualitative differences in exploratively derived topics, i.e., company-provided benefits and governance structures between reviews of companies appearing in Fortune's Best Companies 2014 ranking and 24/7 Wall St.'s 2014 Worst Places to Work ranking were found [37]. Further, the prevalence of exploratively derived employer branding value propositions differed between reviews for best and worst places to work (according to their Glassdoor ranking) and between reviews from former and current employees $[10,11]$.

4.1.3. Uncover the linguistic style of employer reviews. Three studies uncovered the linguistic style of employer reviews. In this vein, significant differences concerning word choice and verbal tone between highest and lowest-rated reviews (i.e., onestar vs. five-star reviews) as well as between the reviews for companies that are ranked best an worst on a B2B-ranking from Brandwatch, which lists the Top200 B2B brands on social media, were found $[33,34]$. More specifically, five-star reviews (vs. one-star reviews) displayed more optimism, embellishment, variety, complexity, commonality, analytical thinking, authenticity, emotional tone, and contained fewer words, but displayed less activity, certainty, realism, insistence, and clout $[12,34]$. Further, reviews for topranked (vs. low ranked) employers displayed more optimism, embellishment, variety, and complexity, but less activity, certainty, and realism [34].

4.1.4. Other. Finally, three studies utilized employer review data not categorizable into previously defined concepts. First, Könsgen et al. analyzed the withincompany deviation of overall ratings and sentiment in a preliminary study [25]. The authors showed that reviews for the same company are rather discrepant and examine the effects of this discrepancy in a subsequent online experiment. Second, M. Huang et al. predicted auditing outcomes (audit fees, issuance of modified going concern opinions, and audit report lag) using employer reviews' satisfaction rating (i.e., overall rating) [20]. They showed that lower ratings increase audit fees, audit report lag length, and firms' likelihood of receiving modified going concern opinions [20]. Third, Robertson et al. compared brand personality perceptions between reviews of highly and lowly ranked firms according to Brandwatch's B2Branking and between highly and lowly rated reviews (according to reviews' overall rating) [36]. They demonstrated that top-ranked (vs. low ranked) employers are perceived as less exciting, rugged, and sincere. Furthermore, high (vs. low) rated (one vs. five stars) reviews displayed more competence, excitement, sincerity, sophistication, but less ruggedness [36].

\subsection{Text Mining Techniques}

4.2.1. Topic modeling. The 16 studies utilizing the textual content of employer reviews applied four distinct approaches to extracting information. First, four studies utilized topic modeling to discover the hidden structure, i.e., topics in employer reviews' textual contents. More specifically, Corritore et al. and Jung and Suh use Latent Dirichlet Allocation (LDA) $[8,24]$. LDA assumes that documents are composed of probabilistically distributed topics, which in turn are composed of probabilistically distributed words [3]. Albeit both studies utilized the same topic model algorithm, each took a different approach regarding pre-processing of the initial text corpus. Jung and Suh [24], before taking further measures such as extracting bigrams, discarded non-noun words as identified by a preliminary part-of-speech tagging. Corritore et al., again before taking additional steps such as trimming nonsense terms, discarded sentences that did not contain the words "culture" or a close synonym [8]. These decisions were based on the results that were to be achieved through the application of LDA. Jung and Suh aimed to identify interpretable and thus coherent job satisfaction factors expressed in employer reviews' textual content [24]. Accordingly, the authors estimated a topic model with 65 topics and clustered them into 30 factors. Corritore et al. on the other hand, used the topic model to develop a measure of interpersonal and intrapersonal heterogeneity based on topic distribution between and within reviews [8]. Therefore, the authors estimated a 500-topic model to estimate conceptually distinct topics. Another class of topic models was utilized by Stamolampros et al. [41, 42]: the Structural Topic Model [STM; 35]. STMs allow accounting for document-level covariates, i.e., review metadata such as reviewers' status (current vs. former), position, department, or industry when 
estimating topics. Thus, STMs relax the restrictive assumption that topics reflect in all documents, i.e., reviews equally [41, 42]. Stamolampros et al. both considered only reviews from tourism and hospitality firms and opted to estimate coherent and interpretable topics to discover job factors relevant for staff in these firms [41, 42]. Stamolampros et al. presented a 10topic model [41]. Stamolampros et al. divided the textual content into positive and negative feedback and presented a 20-topic model [42].

4.2.2. Dictionary-based text analysis. Second, five studies utilized dictionary-based text analysis to extract information from the reviews' textual content. More specifically, three studies applied DICTION [33, 34, 36]. DICTION is a text analysis program developed by communication researchers to identify text dimensions based on their word frequency [40]. In other words, documents are analyzed by counting words assigned to specific dimensions in the document according to pre-defined or user-defined content dictionaries, followed by dividing the count by the total number of words in the document. Pitt et al. utilized DICTION's pre-defined content dictionary and extracted five dimensions from employer reviews that are fundamental for any document: certainty, optimism, activity, realism, and commonality [33]. Further, Pitt et al. extracted these five dimensions and four further dimensions: insistence, embellishment, variety, and complexity [34]. In comparison, Robertson et al. utilized DICTION's capability to estimate the word frequencies of dimension in userdefined content dictionaries and extracted Aaker's five dimensions of brand personality [36]: sincerity, excitement, competence, sophistication, and ruggedness [1]. Duncan et al. used another widely used text analysis program to determine word frequency counts of various dimensions based on predefined or user-defined content dictionaries [12]: LIWC [Linguistic Inquiry and Word Count; 45]. More specifically, the authors focused on LIWC's predefined dictionary and extract four dimensions from employer reviews: analytical thinking, clout, authenticity, and emotional tone [12]. Finally, not using any specific text analysis program, Au et al. used a dictionary-based method to measure employee flexibility in employer reviews' textual content [2]. Specifically, Au et al. developed a word list associated with employee flexibility from literature and extended it using WordNet's thesaurus [2]. Subsequently, the authors calculated a flexibility ratio for each review by counting the total number of words associated with employee flexibility and dividing it by the total number of words per review.
4.2.3. Data-mining software. Third, four studies used data-mining software to extract information from the reviews' textual content. Dabirian et al. both facilitated IBM Watson $[10,11]$. According to Dabirian et al. Watson analyzes content on "(1) parts of speech, including the nouns, verbs, and adjectives that employees used; (2) sequences of words in a sentence and phrase constituents; and (3) sentiment, separating positive and negative expression and phrases" [10, p. 4]. Subsequently, the authors coded IBM Watson's outputs (i.e., expressions and phrases) into content dimensions informed by existing literature. More specifically, Dabirian et al. coded the output into seven employer value propositions: social value, interest value, application value, development value, economic value, management value, work/life balance [10]. Using the same technique, Dabirian et al. added an eighth dimension: brand image [11]. Könsgen et al. also used an IBM technology to identify the sentiment (from -1 (negative) to +1 (positive)) as well as the length in characters of the textual contents of their employer review data: AlchemyAPI [25]. Finally, Ross et al. utilized Leximancer, a text analysis software that creates concept maps (e.g., from word frequencies and common occurrences of words) that allow visual exploration of key concepts and relations in text documents [37].

4.2.4. Individual word frequencies. Fourth, two studies extracted individual word frequencies per employer review and determined their relevance by correlating them with a secondary variable. Accordingly, Storer and Reich identified the words in their employer reviews' pros and cons section that become more common after a minimum wage increase [44]. Further, Moro et al. extracted the ten most frequent nouns in their employer reviews' pros, cons, and management advice section and examined their relevance for reviews' overall ratings using a SVM model [30].

4.2.5. Other. Finally, one study facilitated the length (i.e., number of words) difference between the pros and cons section's comments as an alternative textbased measure of employee satisfaction [16]. Green et al. argued that employees that are satisfied with their employer tend to spend more words in the pro section and fewer words in the contra section of their reviews [16].

\subsection{Data Sources}

4.3.1. Glassdoor. 24 studies used Glassdoor as a data source (https://www.glassdoor.com). Apart from review metadata, i.e., the review's date and the name 
of the reviewed company, Glassdoor's reviews comprise reviewer's status (current vs. former employee), location, type (e.g., full-time, part-time), job title, and tenure. Further, constituting reviews' main source of information, they comprise an overall rating of the employer measured on a one-item fivepoint scale, often used as a proxy for employee satisfaction [e.g., 19], and individual ratings each measured on a one-item five-point scale for work/life balance, culture and values, career opportunities, compensation and benefits, and senior management. Moreover, reviews comprise a business outlook indicator whether employees perceive their employer's business outlook as positive, neutral, or negative, a recommendation indicator (yes, no), and a CEO approval indicator (positive, neutral, negative). Further, textual content is divided into a "Pros", "Cons" and "Advice to Management" section.

4.3.2. Indeed. Two studies used Indeed as a data source (https://indeed.com) [2, 37]. Indeed reviews comprise the following apart from the review date and name of the reviewed employer [2]: reviewer's job title, and location, individual ratings measured on a one-item five-point scale (job/work-life balance, compensation benefits, job satisfaction, management, and job culture), an overall rating as mean of these individual scales, and a free-text comment as well as comments for pros and cons. In this vein, the information provided in Indeed reviews is quite comparable to Glassdoor's review information.

4.3.3. Jobplanet. One study used Jobplanet as a data source (https://www.jobplanet.co.kr/). Jobplanet is one of the most popular review sites in South Korea [24]. Jobplanet delivers the same information as Glassdoor, including an outlook indicator as well as the same individual ratings [24].

4.3.4. Kununu. One study used Kununu as a data source (https://www.kununu.com/) Kununu is one of the most popular review sites in the German-speaking area [25]. Könsgen et al. used Kununu reviews' textual content and overall rating (1 to 5 stars) for their preliminary study, i.e., Study 1 [25]. However, the authors give no further insight into the information provided via Kununu's reviews other than that they comprise a title, a text as well as a rating.

4.3.5. Sample and Matching. Studies in my review set used 1,185 [37] to 1,245,000 [2] unique employer reviews for their analyses. The majority use Compustat data or CRSP data to enrich their review data set with additional variables. Moreover, studies used data from SmartMoney.com, Thomson-Reuters
I/B/E/S First Call, Thomson Reuters Eikon, Thomson Reuters Datastream Infobase, Thomson Reuters Institutional (13f) Holdings, Execucomp, and BoardEx as well as obtained Satisfaction Index (ACSI), and Kinder, Lydenberg, Domini Research \& Analytics (KLD) Social Ratings data. Studies that predicted employee satisfaction also combined employer review data with data not retrieved from a dedicated database, but from a content analysis of mission statements from Fortune 500 companies' websites [6], a survey of firms headquartered in the US and Ireland [32], and state-level information on minimum wage increase [44].

\section{Discussion}

My SLR revealed 28 high-quality peer-reviewed journal articles from a multitude of disciplines that utilize employer review data. My review indicates that online employer reviews are a fast-growing topic in academia. I structured the current research to allow further exploitation of this source of data.

My analysis revealed three major research topics. First, studies that extract information from employer review data to predict firm performance. Specifically, these studies gain information about employee satisfaction and changes in employee satisfaction, insider knowledge, and insights into workplace culture from employer review data. They use this information to predict, e.g., ROA, Tobin's Q, or patent output. Second, studies that explore factors of employee satisfaction. Specifically, these studies explore the role of employee satisfaction factors derived from nonemployer review data, i.e., information on organizations' structure, workplace culture, and financials as well as on policies. Furthermore, studies explore the role of factors derived from employer review data, especially reviews' textual content. Finally, studies that uncover the linguistic style of employer reviews. More specifically, these studies compare the linguistic style of best and worst companies.

Studies across all research topics utilized various text mining techniques to quantify the textual content of reviews. I identified four groups of techniques used so far: topic modeling, dictionary-based text analysis using programs such as DICTION, data-mining using software such as IBM Watson, and extracting individual word frequencies. Regarding data sources, I noticed that Glassdoor data is majorly used and often merged with Compustat and/or CRSP data. I aggregated my findings into a comprehensive research framework (see Figure 3). 


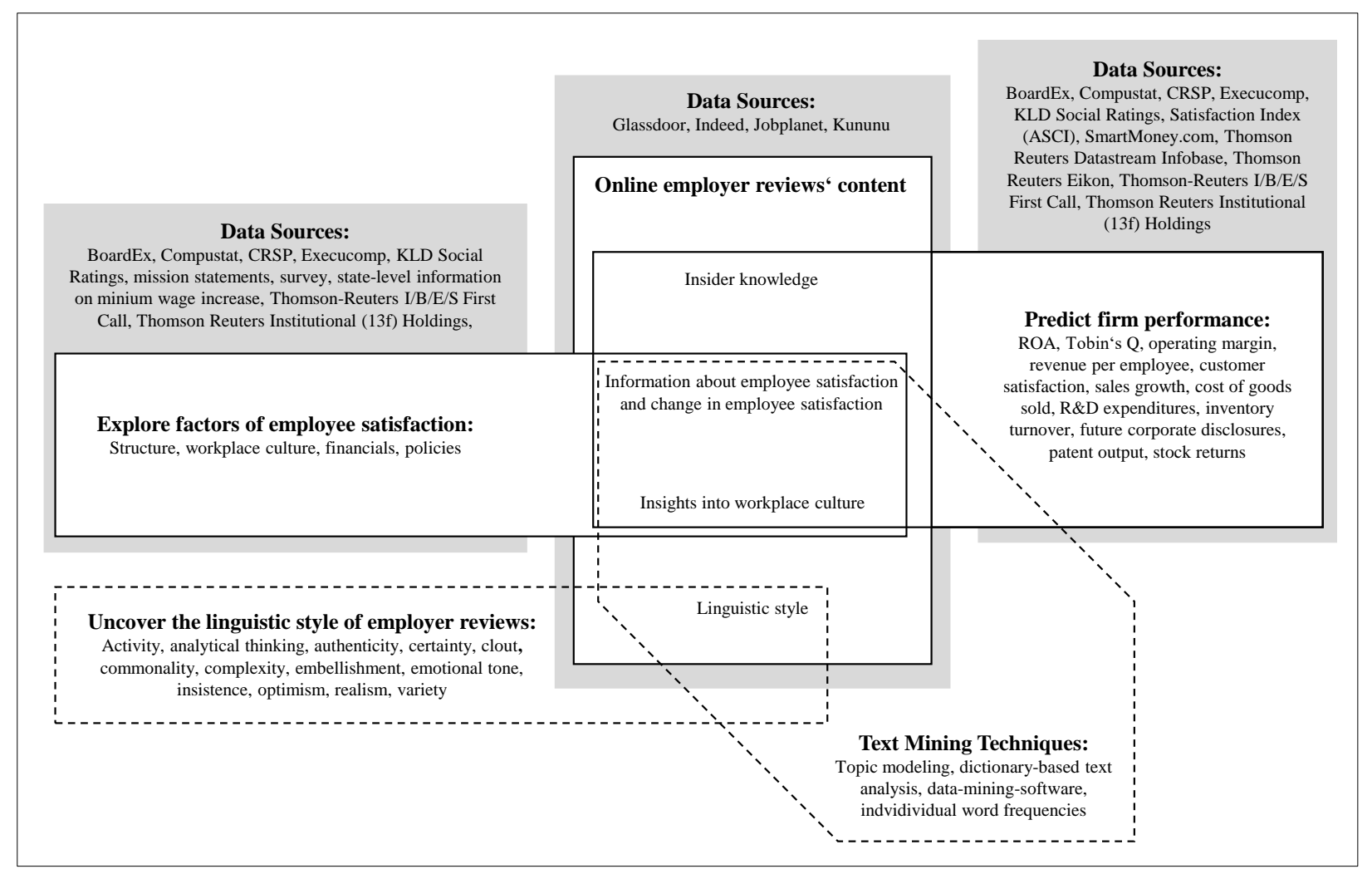

Figure 3. Employer review data research framework

\subsection{Avenues for Future Research}

Based on my SLR, I envision five major research topics to focus on in the next years. First, I see further research opportunities in predicting firm performance from information gained from employer reviews' textual content. Only two studies identified with my SLR gained insight into workplace culture from employer reviews' textual content to predict firm performance [2, 8]. However, constructs developed for computer-aided text analysis such as organizational psychological capital, which is defined as an "organization's level of positive psychological resources: hope, optimism, resilience, and confidence" [27, p. 157] offer great potential for gaining novel inside into workplace cultures from employer review data to predict firm performance.

Second, extending my first point, I see great value for academics and practitioners alike in a theorydriven discovery of employer review's textual content. My SLR indicates that there is a lack of theory-driven analysis of the textual content of online employer reviews. Instead, explorative approaches such as topic modeling or other data mining software are used to quantify the textual content [e.g., 24]. Further, in terms of dictionary-based approaches, dictionaries developed for other contexts were used and thus also represent an explorative approach. While explorative analyses provide great value in understanding online employer reviews' textual contents, the systematic development of dedicated content dictionaries would extend our understanding of online employer reviews derived from theory [39]. For instance, organizational reputation literature provides several suggestions about the type of textual content that should manifest in online employer reviews [see 13].

Third, I see further research opportunities in predicting firm performance with regard to recruiting outcomes. My SLR shows that studies mainly focus on financial indicators of firm performance, such as Tobins' Q, ROA, or revenue per employee. However, given that experimental studies consistently show that employer reviews affect potential employees' attitudes and intentions [14, 25, 28, 43], studying the impact of employer reviews on recruiting indicators, such as quantity and quality of applications, is particularly vital.

Fourth, I see further research necessary in comparing employer reviews with traditional survey instruments. Several authors in my SLR argue that online reviews offer value beyond traditional surveys $[24,41,42]$. However, given the potential impact of self-selection on online employer review data, employer reviews' content should be compared with the results of randomized employee (satisfaction) 
surveys. Although evidence for the generalizability of employer review data on firm-level is also provided by its ability to predict firm performance, a comparison with traditional survey instruments would bring some certainty.

Fifth, further research should be done in exploring the determinants of employer reviews. The SLR studies only give limited insight into what determines the content of employer reviews. Employer review websites do typically not disclose the identity of reviewers within profile information. In this vein, employer review websites opt to offer employees the opportunity to freely judge their employers without fear of legal consequences or other forms of retaliation [22]. Thus, employer review websites differ to some extent in context from product or service review websites. However, such differences serve as a significant distinction between contexts when assessing, e.g., the impact of management responses [7]. Therefore, future research should investigate whether our knowledge of product and service reviews' determinants can be transferred to employer reviews.

\subsection{Limitations}

My literature review is not without limitations. First, I restricted my review to studies that utilize actual employer review data in their analyses. Thus, I exclude studies that investigate the perception of employer reviews, e.g., in regard to organizational attraction [14]. However, understanding employer reviews' perception is an important component in the general understanding of employer reviews. I thus encourage a review of this research stream.

Second, I restricted my review to studies that are listed in the AJG or JCR. This way, I avoid the difficult task of assessing the publications' quality and instead rely on an external quality indicator. However, I exclude a large number of currently circulating working papers that might provide an interesting insight into current research trajectories.

\section{References}

[1] Aaker, J.L., "Dimensions of brand personality", Journal of marketing research, 347-356 34(3), 1997, pp. 347-356.

[2] Au, S.-Y., M. Dong, and A. Tremblay, "Employee Flexibility, Exogenous Risk, and Firm Value", Journal of Financial and Quantitative Analysis, 2019, pp. 1-65.

[3] Blei, D.M., A.Y. Ng, and M.I. Jordan, "Latent Dirichlet Allocation", Journal of Machine Learning Research 3(Jan), 2003, pp. 993-1022.

[4] Briner, R.B., and D. Denyer, "Systematic Review and Evidence Synthesis as a Practice and Scholarship Tool", In
The Oxford Handbook of Evidence-Based Management. 2012.

[5] Cable, D.M., and D.B. Turban, "Establishing the Dimensions, Sources and Value of Job Seekers' Employer Knowledge During Recruitment", Research in Personnel and Human Resources Management 20, 2001, pp. 115163.

[6] Canning, E.A., M.C. Murphy, K.T.U. Emerson, J.A. Chatman, C.S. Dweck, and L.J. Kray, "Cultures of Genius at Work: Organizational Mindsets Predict Cultural Norms, Trust, and Commitment Personality", Personality and Social Psychology Bulletin 00(0), 2019, pp. 1-17.

[7] Chen, W., B. Gu, Q. Ye, and K.X. Zhu, "Measuring and Managing the Externality of Managerial Responses to Online Customer Reviews", Information Systems Research 30(1), 2018, pp. 81-96.

[8] Corritore, M., A. Goldberg, and S.B. Srivastava, "Duality in Diversity: How Intrapersonal and Interpersonal Cultural Heterogeneity Relate to Firm Performance", Administrative Science Quarterly, 2019, pp. 1-36.

[9] Creek, S.A., K.M. Kuhn, and A. Sahaym, "Board Diversity and Employee Satisfaction: The Mediating Role of Progressive Programs", Group \& Organization Management 00(0), 2017, pp. 1-28.

[10] Dabirian, A., J. Kietzmann, and H. Diba, “A great place to work!? Understanding crowdsourced employer branding", Business Horizons 60(2), 2017, pp. 197-205. [11] Dabirian, A., J. Paschen, and J. Kietzmann, "Employer Branding: Understanding Employer Attractiveness of IT Companies", IT Professional 21(1), 2019, pp. 82-89.

[12] Duncan, S.Y., R. Chohan, and J.J. Ferreira, "What makes the difference? Employee social media brand engagement", Journal of Business \& Industrial Marketing 34(7), 2019, pp. 1459-1467.

[13] Etter, M., D. Ravasi, and E. Colleoni, "Social media and the formation of organizational reputation", Academy of Management Review, 2019.

[14] Evertz, L., R. Kollitz, and S. Sü $\beta$, "Electronic wordof-mouth via employer review sites-the effects on organizational attraction", International Journal of Human Resource Management, 2019, pp. 1-30.

[15] Glassdoor, “About Glassdoor”, 2020.

https://www.glassdoor.com/about-us/

[16] Green, T.C., R. Huang, Q. Wen, and D. Zhou, "Crowdsourced employer reviews and stock returns", Journal of Financial Economics 134(1), 2019, pp. 236251.

[17] Hales, J., J.R. Moon, and L.A. Swenson, “A new era of voluntary disclosure? Empirical evidence on how employee postings on social media relate to future corporate disclosures", Accounting, Organizations and Society 68-69, 2018, pp. 88-108.

[18] Huang, K., M. Li, and S. Markov, "What Do Employees Know? Evidence from a Social Media Platform", The Accounting Review 95(2), 2020, pp. 199 226.

[19] Huang, M., P. Li, F. Meschke, and J.P. Guthrie, "Family firms, employee satisfaction, and corporate performance", Journal of Corporate Finance 34, 2015, pp. $108-127$

[20] Huang, M., A. Masli, F. Meschke, and J.P. Guthrie, 
“Clients' Workplace Environment and Corporate Audits", Auditing: A Journal of Practice \& Theory 36(4), 2017, pp. 89-113.

[21] Huang, N., Y. Hong, and G. Burtch, "Social Network Integration and User Content Generation: Evidence from Natural Experiments", MIS Quarterly 41(4), 2017, pp.

1035-1058.

[22] Jackson, A.E., "Can My Boss or Employer Fire Me For Posting on Glassdoor?", Glassdoor, 2016.

https://www.glassdoor.com/blog/can-my-boss-oremployer-fire-me-for-posting-on-glassdoor/ [23] Jing, C., K. Keasey, I. Lim, and B. Xu, "Financial constraints and employee satisfaction", Economics Letters 183, 2019, pp. 108599.

[24] Jung, Y., and Y. Suh, "Mining the voice of employees: A text mining approach to identifying and analyzing job satisfaction factors from online employee reviews", Decision Support Systems 123, 2019, pp. 1-12. [25] Könsgen, R., M. Schaarschmidt, S. Ivens, and A. Munzel, "Finding Meaning in Contradiction on Employee Review Sites - Effects of Discrepant Online Reviews on Job Application Intentions", Journal of Interactive Marketing 43, 2018, pp. 165-177.

[26] Lievens, F., and J.E. Slaughter, "Employer Image and Employer Branding: What We Know and What We Need to Know", Annual Review of Organizational Psychology and Organizational Behavior 3, 2016, pp. 407-440. [27] McKenny, a. F., J.C. Short, and G.T. Payne, "Using Computer-Aided Text Analysis to Elevate Constructs: An Illustration Using Psychological Capital", Organizational Research Methods 16(1), 2012, pp. 152-184.

[28] Melián-González, S., and J. Bulchand-Gidumal, "Worker word of mouth on the internet: Influence on human resource image, job seekers and employees", International Journal of Manpower 37(4), 2016, pp. 709 723.

[29] Melián-González, S., J. Bulchand-Gidumal, and B. González López-Valcárcel, "New evidence of the relationship between employee satisfaction and firm economic performance", Personnel Review 44(6), 2015, pp. 906-929.

[30] Moro, S., R. Ramos, and P. Rita, "What drives job satisfaction in IT companies?", International Journal of Productivity and Performance Management, 2020. [31] Mudambi, S.M., and D. Schuff, "Research Note: What Makes a Helpful Online Review? A Study of Customer Reviews on Amazon.com”, MIS Quarterly 34(1), 2010, pp. 185-200.

[32] O'Reilly, C.A.I., D.F. Caldwell, J.A. Chatman, and B. Doerr, "The Promise and Problems of Organizational Culture: CEO Personality, Culture, and Firm Performance", Group \& Organization Management 39(6), 2014, pp. 595 625.

[33] Pitt, C.S., E. Botha, J.J. Ferreira, and J. Kietzmann, "Employee brand engagement on social media: Managing optimism and commonality", Business Horizons 61(4), 2018, pp. 635-642.

[34] Pitt, C.S., K.A. Plangger, E. Botha, J. Kietzmann, and L. Pitt, "How employees engage with B2B brands on social media: Word choice and verbal tone", Industrial Marketing Management 81, 2019, pp. 130-137.
[35] Roberts, M.E., B.M. Stewart, and E.M. Airoldi, "A Model of Text for Experimentation in the Social Sciences", Journal of the American Statistical Association 111(515), 2016, pp. 988-1003.

[36] Robertson, J., S.L. Ferguson, T. Eriksson, and A. Näppä, "The brand personality dimensions of business- tobusiness firms: a content analysis of employer reviews on social media”, Journal of Business-to-Business Marketing 26(2), 2019, pp. 109-124.

[37] Ross, J.P., M.L. Intindola, and D.M. Boje, "It Was the Best of Times; It Was the Worst of Times: The Expiration of Work-Life Balance", Journal of Management Inquiry 26(2), 2017, pp. 202-215.

[38] Saini, G.K., and I.M. Jawahar, "The influence of employer rankings, employment experience, and employee characteristics on employer branding as an employer of choice", Career Development International 24(7), 2019, pp. 636-657.

[39] Short, J.C., J.C.C. Broberg, C.C. Cogliser, and K.H. Brigham, "Construct Validation Using Computer-Aided Text Analysis (CATA)", Organizational Research Methods 13(2), 2010, pp. 320-347.

[40] Short, J.C., and T.B. Palmer, "The Application of DICTION to Content Analysis Research in Strategic Management", Organizational Research Methods 11(4), 2008, pp. 727-752.

[41] Stamolampros, P., N. Korfiatis, K. Chalvatzis, and D. Buhalis, "Harnessing the 'wisdom of employees' from online reviews", Annals of Tourism Research journal, 2019, pp. 2-5.

[42] Stamolampros, P., N. Korfiatis, K. Chalvatzis, and D. Buhalis, "Job satisfaction and employee turnover determinants in high contact services: Insights from Employees'Online reviews", Tourism Management 75, 2019, pp. 130-147.

[43] Stockman, S., G. Van Hoye, and S. da Motta Veiga,

"Negative word-of-mouth and applicant attraction: The role of employer brand equity", Journal of Vocational Behavior $118,2019$.

[44] Storer, A., and A. Reich, “'Losing My Raise': minimum wage increases, status loss and job satisfaction among low-wage employees", Socio-Economic Review $O(0), 2019$, pp. 1-29.

[45] Symitsi, E., P. Stamolampros, and G. Daskalakis, "Employees' online reviews and equity prices", Economics Letters 162, 2018, pp. 53-55.

[46] Tausczik, Y.R., and J.W. Pennebaker, "The Psychological Meaning of Words: LIWC and Computerized Text Analysis Methods", Journal of Language and Social Psychology 29(1), 2010, pp. 24-54. [47] Webster, J., and R.T. Watson, "Analyzing the Past to Prepare for the Future: Writing a Literature Review.", MIS Quarterly 26(2), 2002, pp. xiii-xxiii.

[48] Wolter, J.S., D. Bock, J.D. Mackey, P. Xu, and J.S. Smith, "Employee satisfaction trajectories and their effect on customer satisfaction and repatronage intentions Employee satisfaction trajectories and their effect on customer satisfaction and repatronage intentions", Journal of the Academy of Marketing Science 47(5), 2019, pp. 815836. 